\title{
Multiple ionisation of rare gases by high-energy uranium ions $\dagger$
}

\author{
H Berg $\ddagger$, R Dörner $\ddagger, C$ Kelbch $\ddagger, S$ Kelbch $\ddagger$, J Ullrich $\ddagger, S$ Hagmann§, \\ P Richard§, H Schmidt-Böcking§, A S Schlachter\|, M Prior\|, \\ H J Crawford\|, J M Engelage $\|$, I Flores $\|$, D H Loyd $\uparrow$, J Pedersen ${ }^{+}$ \\ and R E Olson ${ }^{\#}$ \\ $\ddagger$ Institut für Kernphysik, Universität Frankfurt, D6000, Frankfurt, Federal Republic of \\ Germany \\ § Kansas State University, Manhattan, Kansas 66506, USA \\ || Lawrence Berkeley Laboratory, Berkeley, California 94720, USA \\ T Angelo State University, San Angelo, Texas 76909, USA \\ + University of Aarhus, DK 8000 Aarhus, Denmark \\ \# University of Missouri-Rolla, Rolla, Missouri 65401, USA
}

Received 13 May 1988, in final form 18 July 1988

\begin{abstract}
Multiple ionisation cross sections $\sigma(q)$ for the production of recoil ions in charge state $q$ by $120 \mathrm{MeV} \mathrm{u}^{-1} \mathrm{U}^{90+}$ impact have been measured for $\mathrm{Ne}$, Ar and $\mathrm{Kr}$ targets using a recoil-ion-projectile-electron triple-coincidence technique. The data are compared with previously measured cross sections for U-ion impact in the projectile energy $\left(E_{\mathrm{P}}\right)$ range from 1.4 to $420 \mathrm{MeV} \mathrm{u}^{-1}$. For low recoil-ion charge states $\sigma(q)$ decreases over the whole $E_{\mathrm{P}}$ range somewhat more slowly than $1 / E_{\mathrm{P}}$, whereas for higher recoil-ion charge states $\sigma(q)$ reaches a maximum at about $10-15 \mathrm{MeVu}^{-1}$. The data are nicely described by parameter-free $n$-body Classical Trajectory Monte Carlo (nCTMC) calculations. The calculations indicate the importance of accounting for the Auger events in the description of the multiple ionisation process. Because of the smooth $E_{\mathrm{P}}$ dependence of $\sigma(q)$ towards high $E_{\mathrm{P}}$, the development of a recoil-ion source using a primary $\mathrm{U}$-ion beam in a storage ring for the production of high charge state recoil-ions appears to be feasible.
\end{abstract}

\section{Introduction}

Multiple ionisation of target atoms by fast heavy-ion impact has attracted much interest in recent years. The development of new time-of-flight techniques by different groups (Cocke 1979, Groh et al 1981, Schlachter et al 1981, Hvelplund et al 1980, Damsgaard et al 1982, Kelbch et al 1985, Müller et al 1986) made measurements of differential cross sections for multiple ionisation possible. Theory (Olson 1987, Horbatsch 1986, Horbatsch and Dreizler 1986) also has achieved a breakthrough in describing the many-particle interactions in such encounters. Nevertheless, many aspects of the multiparticle interaction in encounters associated with large perturbation still remain unsolved: can the electrons be treated as independent particles, leading to binomial statistics with respect to the degree of multiple ionisation, or have electron-electron correlation effects a measurable influence on the $\sigma(q)$, where $q$ is the final charge state

† Supported by: Bundesministerium für Forschung und Technologie (BMFT) under grant 060F/73 (741336476), Gesellschaft für Schwerionenforschung (GSI), NATO under grant RG85/0501 and Department of Energy (DOE). 
of the target recoil ion? Another problem is the interaction potential: is it similar to a Thomas-Fermi interaction, or do polarisation and deformation of the target electron cloud influence the collision dynamics of both the electrons and the heavy nuclei? All these effects determine the total multiple ionisation cross section $\sigma(q)$ and are very difficult to treat in a many-particle collision theory. By necessity, theoretical approaches describing the many-electron Coulomb interaction are simplified by several approximations. The theoretical approach of Horbatsch (1986) and Horbatsch and Dreizler (1986) uses an independent-electron model, i.e. no interactions between the electrons are considered. This method provides a numerical solution to the Vlasov equation for the three-body system, i.e. projectile, nucleus and electron. An initial phase space distribution is chosen to reproduce the static properties of the neutral target. Another method (Olson 1987) provides a classical solution to a model, which includes all the target electrons distributed within a microcanonical distribution, but also no electronelectron interaction. This classical model leads to interesting predictions of ionisation phenomena for high projectile charge states in fast collisions: due to the strong two-centre nuclear Coulomb force interaction the electron emission can be very anisotropic in comparison with ionisation by protons.

One goal of our systematic investigation of multiple ionisation processes is to obtain $\sigma(q)$ for U-ion impact over a wide projectile energy range $\left(E_{\mathrm{p}}\right)$. In particular, the dependence of $\sigma(q)$ on the charge state at different $E_{\mathrm{p}}$ is a measure of $n$-body effects. Besides this interest in the understanding of the fundamental mechanisms of multiple ionisation, the application of multiple ionisation as an efficient tool for the production of highly charged very slow $(<10 \mathrm{eV})$ recoil ions by fast ions in a heavy-ion storage ring is of considerable importance for atomic physics. U-ion beams of lower energy might have higher ionisation power, particularly for the outer electrons. However, fast beams are needed in a storage ring, as beam loss due to charge exchange decreases strongly for higher $E_{\mathrm{P}}$ and has to be sufficiently small to ensure long ion storage times. Therefore, the multiple ionisation of noble gases for very high energy $\left(120 \mathrm{MeV} \mathrm{u}^{-1}\right)$ $\mathrm{U}$-ion impact was investigated in the present experiment. As will be discussed below, non-perfect beam focusing and vacuum conditions in the beam line of the Bevalac accelerator and background ionisation in walls, etc, made the detection of recoil ions in high charge states, produced in a gas target with densities below a few $\mathrm{ng} \mathrm{cm}^{-2}$, extremely difficult. A triple coincidence between recoil ions, projectile and electrons was essential to suppress, at least partially, the large ionisation yield of the background radiation.

\section{Experiment}

The measurement of recoil-ion production for $\mathrm{Ne}, \mathrm{Ar}$ and $\mathrm{Kr}$ targets by $120 \mathrm{MeV} \mathrm{u}^{-1} \mathrm{U}^{90+}$ was performed at the Bevalac accelerator at the Lawrence Berkeley Laboratory. The charge-state-selected $\mathrm{U}^{90+}$ beam was focused in the gas-target region to a beam spot of about 5-6 mm (FWHM). The U-ion beam passed a windowless thin gas target $\left(5 \times 10^{-5}\right.$ to $4 \times 10^{-4}$ Torr) and produced the recoil ions and electrons (see figure 1). The projectiles were detected about $3 \mathrm{~m}$ downstream of the target by a fast scintillation detector. Recoil ions were extracted and accelerated perpendicular to the direction of the U-ion beam in an electrostatic field (about $100 \mathrm{~V} \mathrm{~cm}^{-1}$ ) over a length of $17 \mathrm{~cm}$, then drifted in a quasi-field-free region, and could be deffected magnetically onto a two-dimensional position-sensitive channel-plate detector to separate low and 


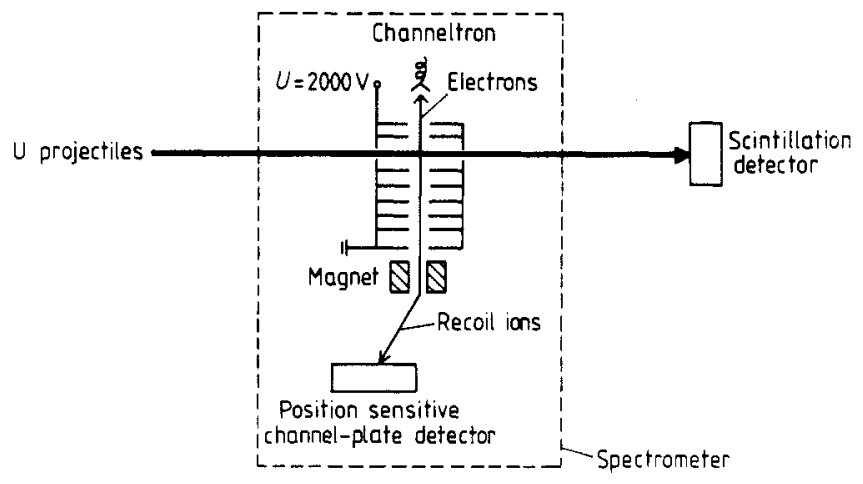

Figure 1. Experimental set-up.

high charge state recoil ions. Electrons emitted in the collision were accelerated in the opposite direction in the same field and were detected by a channeltron detector. The voltages on several slits between the extraction window and the channeltron could be varied independently to increase the electron-detection efficiency. The recoil-ion charge state was determined by a time-of-flight measurement (TOF), since the flight time $t_{\mathrm{R}}$ from the centre of the gas target to the detector is proportional to $1 / \sqrt{ } q$. The spectrometer was designed to use a large-diameter $U$ beam $(\simeq 10 \mathrm{~mm} \varnothing)$. The gas-target region had an opening $>20 \mathrm{~mm}$, to allow transmission of the projectiles without direct impact on the apparatus. The dimensions of the recoil-ion extraction and drift region were designed to obtain optimum time focusing. The absolute time resolution $\tau$ was better than $4 \mathrm{~ns}$ and the relative resolution $\tau / t_{\mathrm{R}} \simeq 10^{-3}$. Such time resolution with a large-diameter beam was necessary to suppress random background events. To obtain an even better suppression of the background, a triple coincidence with the electrons from the ionisation event was implemented. The electronic set-up is shown in figure 2. The events were stored on tape in list mode using the VME system (McParland and Bronson 1987).

A very large background was observed, predominantly associated with recoil-ion charge states $q=1$ and $q=2$. To reduce the triple-coincidence efficiency for these low

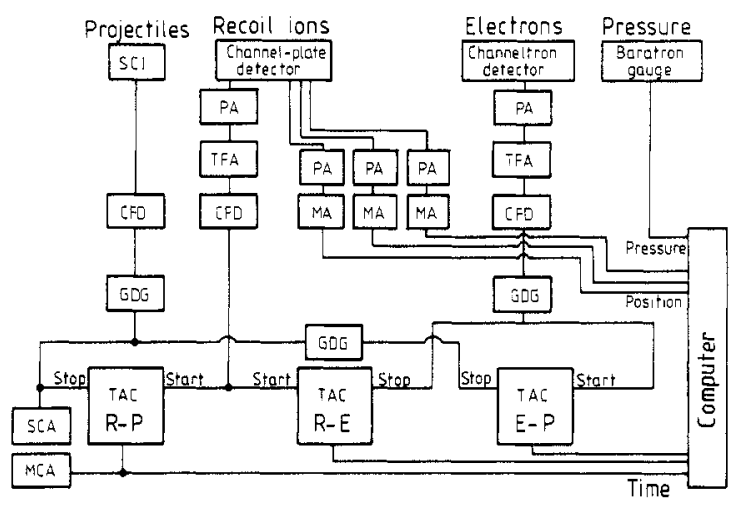

Figure 2. Schematic diagram of electronic arrangement. SCI: scintillator; GDG: gate and delay generator; PA: preamplifier; TAC: time-to-amplitude converter; MA: main amplifier; SCA: scaler; TFA: timing filter amplifier; MCA: multichannel analyser; CFD: constant fraction discriminator. 
recoil-ion charge states, the efficiency to detect one electron was set to about three per cent. For a recoil ion in charge state $q, q$ electrons are emitted to the continuum; the detection probability $P_{\mathrm{e}}(q)$ is proportional to $1-\left(1-P_{c}(q=1)\right)^{q}$. Thus for $q=10+$, a triple-coincidence efficiency of about $30 \%$ (see figure 3 ) could be obtained.

Figure 4 shows a recoil-ion-projectile coincidence spectrum for $120 \mathrm{MeV} \mathrm{u}^{-1} \mathrm{U}^{90+}$ impact on $\mathrm{Ar}$ in comparison with a spectrum for $15.5 \mathrm{MeV} \mathrm{u}^{-1} \mathrm{U}$ on Ar measured with a well collimated beam at the GSI Unilac (Kelbch et al 1985). The relative contribution of random coincidences for the $120 \mathrm{MeV} \mathrm{u}^{-1} \mathrm{U}$-ion impact is orders of magnitude higher than at the lower energies. Triple-coincidence data are shown in figure 5 (recoil-ion-projectile coincidence against recoil-ion-electron coincidence). The diagonal (III) represents the real triple-coincident events, where, for each recoil ion, the projectile and electrons released in the same event were detected. Regions I and

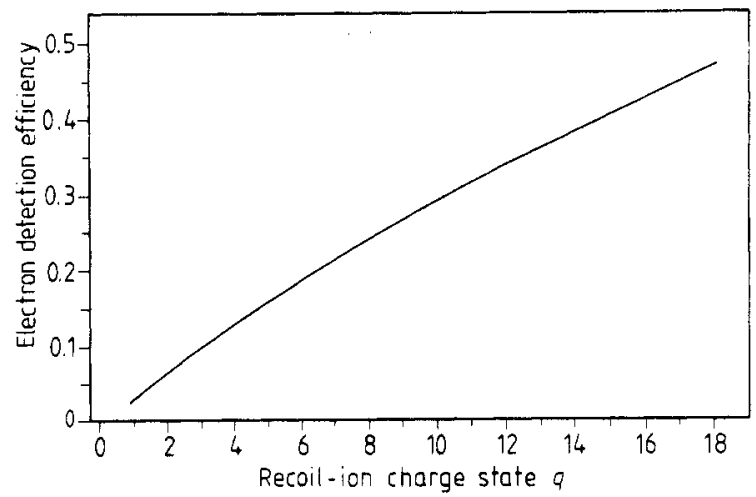

Figure 3. Calculated electron-detection efficiency as a function of the recoil-ion charge state $q$.

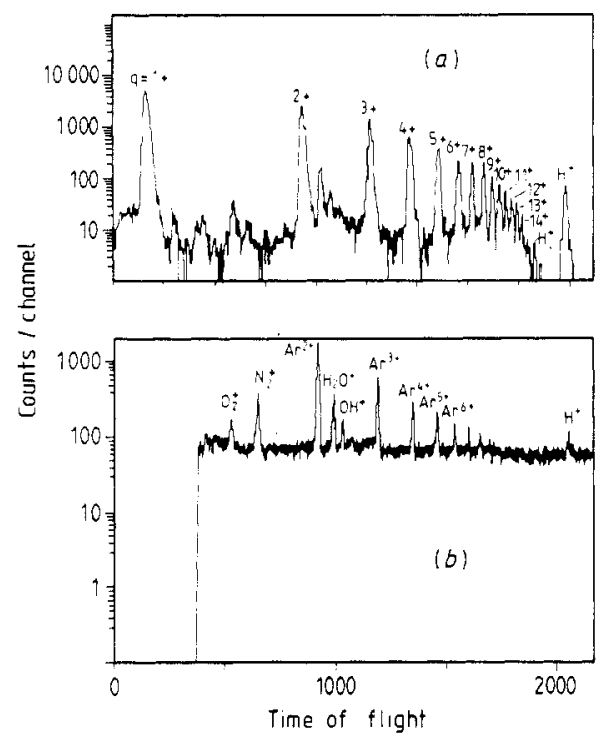

Figure 4. Comparison of recoil-ion-projectile time-of-flight spectra for (a) 15.5 $\mathrm{MeVu}^{-1} \mathrm{U}^{75+}$ and (b) $120 \mathrm{MeVu}^{-1} \mathrm{U}^{90+}$ on Ar collisions. 


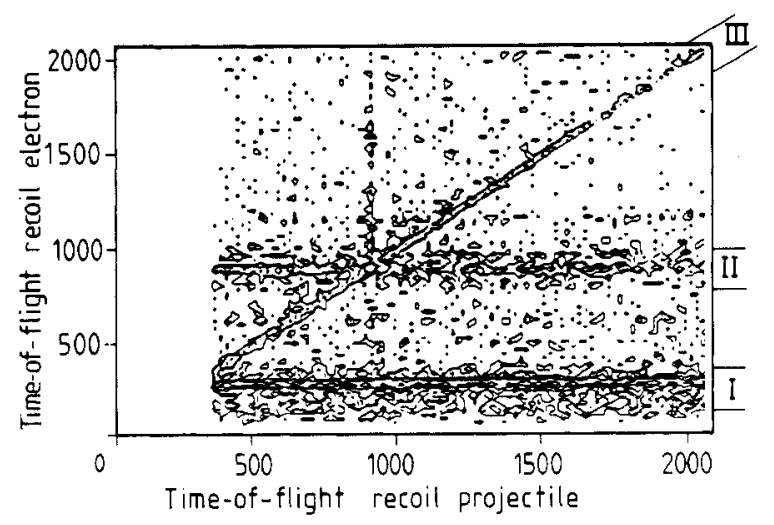

Figure 5. Two-dimensional contour plot of recoil-ion-projectile against recoil-ion-electron time-of-flight spectra for $120 \mathrm{MeV} \mathrm{u}^{-1} \mathrm{U}^{90+}$ on Ar. The regions I, II and III are explained in the text.

II show the recoil-ion-electron coincidence for $q=1+$ and $2+$, for which the corresponding true projectile has not been detected (random events in the projectile detection). These recoil-ion-electron coincidences are produced by the very large $\mathrm{x}$-ray and fast electron background flux, which ionises target-gas atoms in the whole target region, whereas 'real' recoil ions are merely produced in the track of the projectiles. The width of the time peaks of $q=1+$ and $2+$ recoil ions in the recoil-ion-electron ToF spectrum is much larger than in the recoil-ion projectile coincidence, indicating that these recoil ions are produced over the whole extraction length of the spectrometer. Figure 6 shows the dependence of the background yield on the target-gas pressure, from which we can deduce how these background $x$-rays and electrons are created. The linear dependence clearly indicates that these events are indeed produced by single collisons of $\mathrm{x}$-rays and electrons with target atoms, rather than by double collision processes in the target gas. For a double collision (first step: $x$-ray and electrons are produced by the $U$ ions in the gas; second step: $q=1,2$ ionisation of gas atoms by these x-rays and electrons) the yield would be proportional to the square of the pressure. Because of the long beam transport lines $(>40 \mathrm{~m})$ and a beam line pressure greater than $10^{-5}$ Torr, a small fraction $(0.1-1 \%)$ of the projectile beam underwent charge exchange and gave rise to a wider halo of the beam due to different trajectories in the dipole and quadrupole magnets. Depending on the focusing conditions this fraction could be strongly reduced,

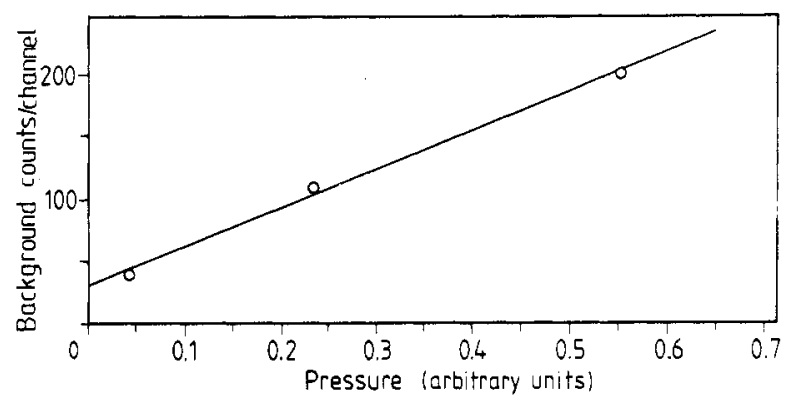

Figure 6. Gas-target pressure dependence of the 'background' yield in the recoil-ion $q=1+$, $2+$ charge states obtained in the recoil-ion-projectile coincidence. 
but was always present even under the best beam conditions. If only 5-10 $\mathrm{U}$ ions per beam spill (every $6 \mathrm{~s}$ ) hit the wall in the vicinity of the apparatus, millions of high-energy $\mathrm{x}$-rays and electrons are produced, which ionise and produce a large number of low-charge-state recoil ions in the thin gas target.

The background can be strongly reduced by setting a diagonal window (III) in figure 5. Integration of the relevant recoil-ion charge-state peaks and correction with the electron detection efficiency yields the number of true events per charge state $N(q)$. To obtain absolute cross sections $\sigma(q)$ the total number of projectiles $N_{\mathrm{P}}$ was measured. The target gas pressure $p$ was measured with a Baratron capacitive manometer. The recoil-ion detection efficiency, $\varepsilon \Delta \Omega_{\operatorname{Rec}} / 4 \pi$ ( $\varepsilon$ efficiency of the channel-plate detector), multiplied by the effective gas-target thickness $\Delta x$ monitored with a Baratron gauge, was measured independently at the $2.5 \mathrm{MV}$ accelerator at the University of Frankfurt, using known charge-exchange cross sections for $2 \mathrm{MeV} \mathrm{u}^{-1} \mathrm{Ne}^{2+}$ on $\mathrm{Ne}$ (Ullich et al 1986). This normalisation was necessary, because the pressure profile in the target region was not exactly known due to the windowless gas-target system. The $\sigma(q)$ are calculated using equation (1):

$$
\sigma(q)=N(q) 4 \pi /\left(N_{\mathrm{P}} \Delta x p \varepsilon \Delta \Omega_{\mathrm{Rec}}\right) .
$$

Figure 7 shows the recoil-ion distribution on the two-dimensional channel-plate detector indicating that the recoil-ion detector itself $(40 \mathrm{~mm}$ diameter) and the recoil-ion extraction system yielded a nice recoil-ion beam spot and thus a controlled detection efficiency.

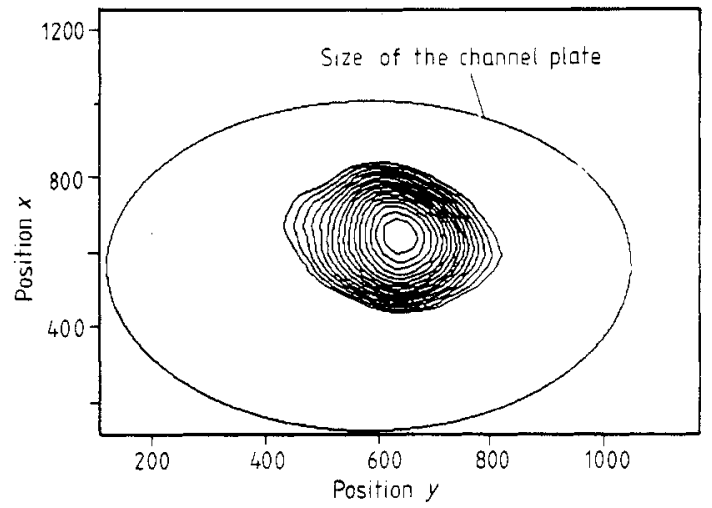

Figure 7. Two-dimensional position distribution of recoil ions on the recoil-ion channel plate detector. The $z$ scale is logarithmic.

\section{Data and discussion}

Figure 8 presents the cross sections for recoil-ion production in $\mathrm{Kr}, \mathrm{Ar}$ and $\mathrm{Ne}$ targets for $120 \mathrm{MeV} \mathrm{u}^{-1} \mathrm{U}^{90+}$ impact. The error bars show only the statistical errors; the error in the absolute normalisation is estimated to be about $30 \%$. The data were obtained at a pressure of $2-3 \times 10^{-4}$ Torr. For higher pressure $\left(4.5 \times 10^{-4}\right.$ Torr $)$ a $50 \%$ reduction of the highest charge-state rate was observed, indicating charge capture of the slow, highly charged recoil ions in the extraction region. Therefore, even the lower pressure data may somewhat $(30-50 \%)$ underestimate the 'true' cross sections for high $q>10$. 


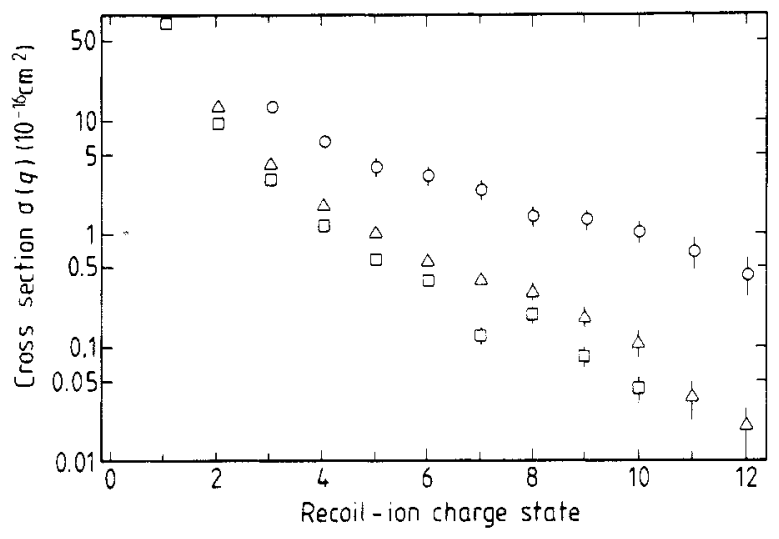

Figure 8. Measured total cross sections $\sigma(q)$ for $120 \mathrm{MeVu}^{-1} \mathrm{U}^{90+}$ on $\mathrm{Ne}(\square), \operatorname{Ar}(\triangle)$ and $\mathrm{Kr}(\mathrm{O})$.

Figure 9 presents all available Ar ionisation data measured for U-ion impact from 1.4 to $420 \mathrm{MeV} \mathrm{u}^{-1}$ as a function of the projectile energy (Kelbch et al 1985, 1986, Müller et al 1986). Note that the normalisation of the data from Kelbch et al (1985), Richard et al (1985) and Ullich et al $(1984,1987)$ has been twice remeasured with improved techniques, because the pressure meter (Baratron gauge) used in the first measurement (Kelbch et al 1985) had to be recalibrated. The single capture channel in the previous experiment also included small-angle and slit-scattering contributions due to a non-perfect projectile charge-state separation. The two new independent measurements with a $27 \mathrm{~cm}$ long gas cell yielded for the single electron capture cross section $\sigma\left(n=55, n^{\prime}=54,5.9 \mathrm{MeVu}^{-1}\right)=7.96$ and $8.8 \times 10^{-17} \mathrm{~cm}^{2}$. Therefore, all $\sigma(q)$ in Kelbch et al (1985), Richard et al (1985) and Ullrich et al $(1984,1987)$ have to be divided by the factor 3.9. Furthermore, figure 3 in Kelbch et al (1985) and Richard

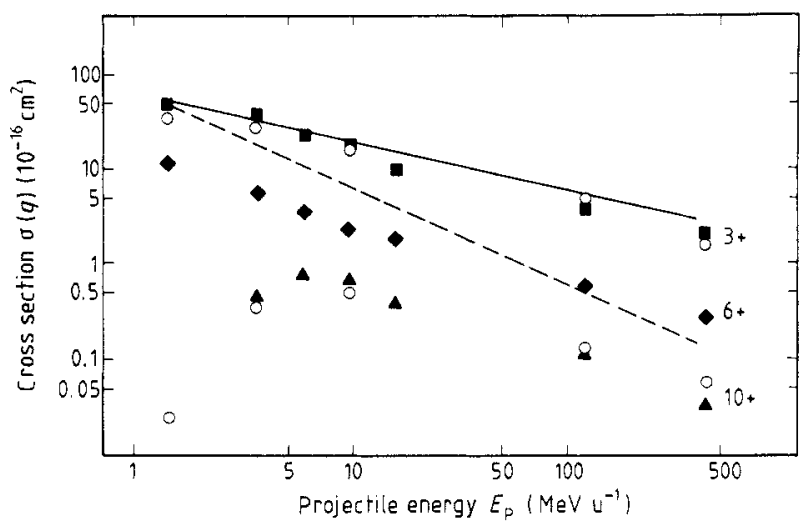

Figure 9. Projectile energy $\left(E_{\mathrm{p}}\right)$ dependence of the multiple ionisation cross sections $\sigma(q)$ for $\mathrm{Ar}$ (the full symbols represent the measured data for $q=3+, 6+, 10+$ ). The $U^{n+}$ charge states were $n=44$ at $1.4 \mathrm{MeVu}^{-1}, n=50$ at $3.9 \mathrm{MeVu}^{-1}, n=55$ at $5.9 \mathrm{MeVu}^{-1}, n=65$ at $9.4 \mathrm{MeVu}^{-1}, n=75$ at $15.5 \mathrm{MeVu}^{-1}, n=90$ at $120 \mathrm{MeVu}^{-1}$ and $n=91$ at $420 \mathrm{MeV} \mathrm{u}^{-1}$. The full line shows the relative $1 / \sqrt{ } E_{\mathrm{p}}$ and the broken line the $1 / E_{\mathrm{p}}$ dependence normalised to the experimental value $\sigma\left(q=3,1.4 \mathrm{MeVu}^{-1}\right)$. The open circles represent $n \mathrm{CTMC}$ calculations for $q=3+$ and $q=10+$. 
et al (1985) show the wrong scales (factor of 10 too small). The $\mathrm{U}$ charge states were $n=50$ at $3.6 \mathrm{MeVu}^{-1}, n=55$ at $5.9 \mathrm{MeV} \mathrm{u}^{-1}, n=65$ at $9.4 \mathrm{MeV} \mathrm{u}^{-1}$, and $n=75$ at $15.5 \mathrm{MeV} \mathrm{u}^{-1}$.

The circles in figure 9 represent $n$ CTMC calculations for $q=3$ and $q=10$. All calculated values shown were determined via the nCTMC method (Olson 1987), where the Ar K-, L- and M-shell electrons are explicitly included in the calculations. Auger effects are taken into account by calculating the total electronic energy of the residual Ar ion after the collision and assuming that the relaxation to the ground-state configuration occurs with $100 \%$ branching by multiple Auger decay. The Auger process is significant and is found to increase the final charge state of the recoil ions by more than one charge state.

All $\alpha(q)$ for $q<8$ decrease smoothly with a slope between $1 / v_{\mathrm{p}}$ and $1 / E_{\mathrm{p}}$ for increasing $E_{\mathrm{P}}$. Figure 9 also shows the relative $1 / E_{\mathrm{P}}$ and $1 / v_{\mathrm{P}}$ dependencies (broken and full lines). This behaviour is not expected from first-order perturbation theory since for lower $q$ the energy dependence is predicted to be approximately proportional to $\left(1 / E_{\mathrm{P}}\right)^{q}$ for small perturbation (Inokuti 1971). For $q>10$ the cross sections $\sigma(q)$ peak at $E_{\mathrm{P}} \geqslant 10 \mathrm{MeVu}^{-1}$. The maximum of the $\sigma(q)$ shifts towards higher $E_{\mathrm{P}}$ with increasing $q$. Therefore we expect that the $\sigma(q=17,18)$ cross sections for Ar continue to increase for $E_{\mathrm{P}}>15.5 \mathrm{MeV} / \mathrm{u}$ and may reach a maximum at $E_{\mathrm{P}} \simeq 20-40 \mathrm{MeV} \mathrm{u}^{-1}$.

Figure 10 presents a comparison of $n$ CTMC calculations by Olson with the measured $120 \mathrm{MeV} \mathrm{u}^{-1}$ data for U-ion impact on Ar. The full curve represents the calculation, which includes autoionisation processes occurring after the collision. The broken curve shows the theoretical $\sigma(q)$ before autoionisation. The comparison of both values indicates that a considerable fraction of low-energy electrons will be produced in these collisions by Auger cascades occurring after the collision. The number of Auger electrons can be deduced from the theory but not from figure 10 directly, since a shift in the absolute height of the cross section as well as in the charge-state scale is involved. It is apparent from figure 9 that multiple ionisation cross sections are large compared with the dimension of the $\mathrm{Ar}$ atom. Ionisation probabilities exceed the $10 \%$ level by far (Ullrich et al 1988) at all projectile energies and thus, perturbation techniques are inappropriate to describe the collision mechanisms. The energy dependence of the $\sigma(q)$ cross sections predicted by the nCTMC theory is in good agreement

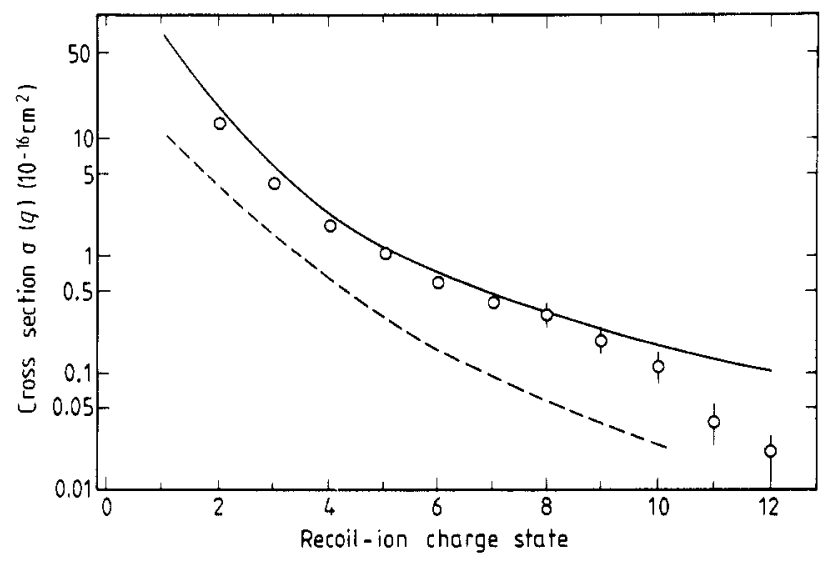

Figure 10. Comparison of measured $\sigma(q)$ with nCTMC calculations; 0 , experiment; autoionisation included; - - -, autoionisation not included. 
with the experiment. Considering the complexity of this many-body process, the overall agreement is excellent, but still considerable information on scattering dynamics is to be gained from further studies of these collisions. In particular the extrapolation of calculated cross sections towards highest $q$ and $E_{\mathrm{P}}$ is risky, because the contribution of autoionisation processes after the ionisation and excitation of a shell is not yet exactly known. Therefore it is necessary to measure the cross sections up to $q=Z_{\mathrm{T}}$. The comparison with the calculated values is a crucial test for the correct implementation of Auger processes into theoretical models. Another problem is whether the classical electron picture is valid towards highest $E_{\mathrm{P}}$, or whether wavepackets are needed to describe the electrons. To answer this question, it is essential to obtain data for highest $q$ and $E_{\mathrm{P}}$.

\section{Prospects for a recoil-ion source at an U-ion storage ring}

An U-ion storage ring beam provides an unique ionisation power to produce very cold highly charged ions (Ullrich et al 1984, 1987).

From the data and $n$ CTMC calculations it is clear that outer-shell ionisation decreases towards high $E_{\mathrm{P}}$, whereas inner-shell ionisation reaches a maximum at $E_{\mathrm{P}} \simeq$ $2 E_{\mathrm{B}} M_{\mathrm{P}} / m_{\mathrm{E}}$, where $E_{\mathrm{B}}$ is the electron binding energy and $M_{\mathrm{P}}$ and $m_{\mathrm{E}}$ are the masses of projectile and electron respectively. The combination of outer- and inner-shell ionisation finally reduces $\sigma\left(q \rightarrow Z_{\mathrm{T}}, E_{\mathrm{P}}\right)$ for high-energy U-ion beams ( $E_{\mathrm{P}}>$ $\left.100 \mathrm{MeVu}^{-1}\right)$. Nevertheless, the data from figure 11, where the ratios $\sigma\left(q, E_{\mathrm{p}}\right) / \sigma\left(q, E_{\mathrm{p}}=3.6 \mathrm{MeVu}^{-1}\right)$ normalised to the charge state $\mathrm{Ar}^{3+}$ are plotted, and the data from Kelbch et al (1985) and Richard et al (1985), where the absolute cross section up to $q=18+$ for $15.5 \mathrm{MeV} \mathrm{u}^{-1} \mathrm{U}^{75+}$ on $\mathrm{Ar}$ are shown, allow an extrapolation towards $\sigma\left(\mathrm{U}^{90+} \rightarrow \mathrm{Ar}, q=17, E_{\mathrm{P}}=120 \mathrm{MeV} \mathrm{u}^{-1}\right)$. The relative $q$ dependence of $\sigma(q)$ is almost identical for $3.6,120$ and $420 \mathrm{MeV} \mathrm{u}^{-1}$. Since for the inner shell we expect even higher ionisation cross sections at 120 than at $3.6 \mathrm{MeV} \mathrm{u}^{-1}$, the ratio in figure 11 for higher charge states may be larger than 1 and may even approach, for $E_{\mathrm{p}} \simeq$ $50 \mathrm{MeVu}^{-1}$, those for 9.4 and $15.5 \mathrm{MeV} \mathrm{u}^{-1}$. Thus we estimate by extrapolation that

$$
\frac{\sigma\left(\mathrm{Ar}, q=17+, E_{\mathrm{P}}=120 \mathrm{MeV} \mathrm{u}^{-1}\right)}{\sigma\left(3+, 120 \mathrm{MeVu}^{-1}\right)}=\frac{\sigma\left(17+, 15.5 \mathrm{MeVu}^{-1}\right)}{\sigma\left(3+, 15.5 \mathrm{MeVu}^{-1}\right)} \text {. }
$$

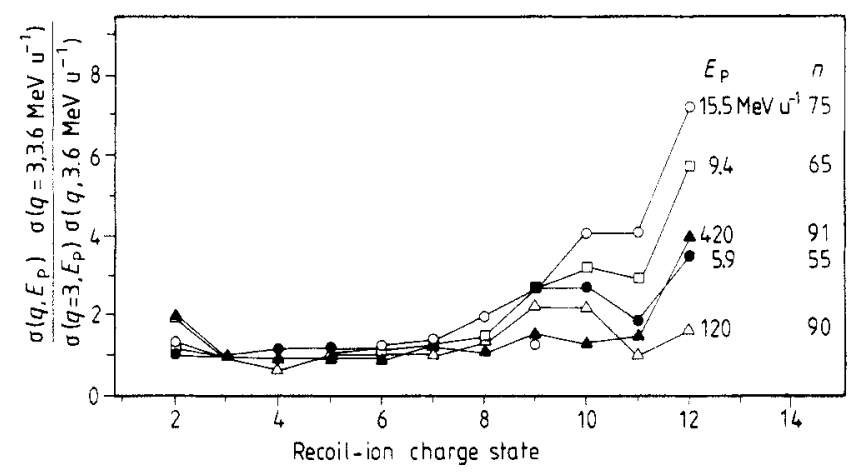

Figure 11. $\left[\sigma\left(q, E_{\mathrm{P}}\right) / \sigma\left(q=3, E_{\mathrm{p}}\right)\right] /\left[\sigma\left(q, 3.6 \mathrm{MeVu}^{-1}\right) / \sigma\left(q=3,3.6 \mathrm{MeVu}^{-1}\right)\right]$ cross section ratio for $\mathrm{U}^{n+}$ on Ar as function of the recoil-ion charge state for various values of $E_{\mathrm{p}}$ $\left(\mathrm{MeVu}^{-1}\right)$. 
For $\sigma\left(q=17+, E_{\mathrm{P}}=120 \mathrm{MeV} \mathrm{u}^{-1}\right)$ we estimate a value of the order of $10^{-18} \mathrm{~cm}^{2}$.

For $5 \times 10^{10} \mathrm{U}$ ions stored in the storage ring, $2 \times 10^{6}$ cycles per second and a gas-target pressure of $3 \times 10^{13} \mathrm{Ar}$ atoms $/ \mathrm{cm}^{2}$, the number of $\mathrm{Ar}^{17+}$ ions can be estimated using the extrapolated cross section to be $3 \times 10^{12}$ ions $/ \mathrm{s}$, or approximately $8 \mu \mathrm{A}$. This recoil-ion beam is known to have a very small energy spread since the recoil ions are produced with very small recoil-ion energy (Olson et al 1987). Since at this target pressure the $120 \mathrm{MeV} \mathrm{u}^{-1} \mathrm{U}^{90+}$ beam will undergo several electron capture and loss processes per second, the $U$ beam can be stored with acceptable storing periods ( $\sim$ minutes) only if the storage ring is capable of multiple charge transport. Since capture and loss are in equilibrium at $n=90$ the storage time can be enhanced strongly.

The recoil-ion yield can be improved by the construction of a solenoidal ion trap, where successive ionising collisions become possible. Pre-ionisation of the target considerably enhances the high-charge-state cross sections under storage ring conditions. The enhancement is energy dependent and is found (Olson 1987) to amount to only a factor of 1.3 at $9.4 \mathrm{MeV} \mathrm{u}^{-1}$. However, it rises to $\sim 10^{3}$ at $120 \mathrm{MeV} \mathrm{u}^{-1}$ and $\sim 10^{5}$ at $420 \mathrm{MeVu}^{-1}$. The reason for this dependence is the rapidly decreasing probability with increasing energy for removing $M$-shell electrons. Using trapped ions as target, e.g. in an ECR trap, pre-ionisation of the target will greatly increase the hydrogenic and fully stripped $\mathrm{Ar}^{17+}$ and $\mathrm{Ar}^{18+}$ yields at storage ring energies.

In a solenoidal ECR trap, a two-stage device (figure 12) could be additionally used to efficiently remove the outer electrons of the target atom. The first ECR stage would produce low-charge-state ions, which are injected into the second stage, where they are further ionised by the $R F$ and the $U$ beam. As the ions are drifting in a solenoid (length $\sim 1 \mathrm{~m}$, trapping time several hundred $\mathrm{ms}$ ), the efficiency for the production of very high charge states increases due to two factors. Firstly, the outer-shell electrons are already removed, which enhances the ionisation power of the U-ion beam and, secondly, the long trapping time in the solenoid increases the probability of multiple interactions with the $U$ beam. For an ion trap device the charge exchange of the primary beam is reduced and multiple charge transport may not be necessary. On the basis of measured $\sigma(q)$, we believe that it is possible to reach a yield of $\mathrm{Ar}^{17+}$ in the order of several tens of $\mu \mathrm{A}$. This indicates that such a combination could also provide an excellent source for the production of very highly charged $\mathrm{Kr}$ and $\mathrm{Xe}$ recoil ions.

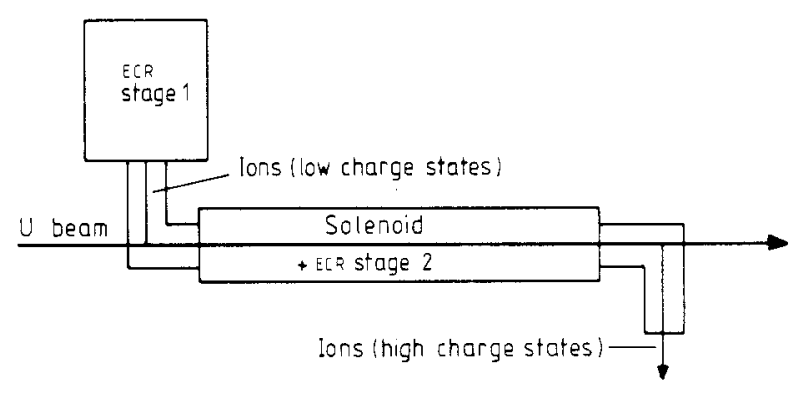

Figure 12. Scheme of a possible recoil ion source at a fast U-beam storage ring. Low $(q=1+, 2+)$ charged recoil-ions are produced in the first stage of a two-stage ECR ion source and injected in the second stage. In the strong solenoidal field the recoil ions are trapped for several $100 \mathrm{~ms}$. In this second stage the primary beam can further ionise the trapped recoil ions by successive collisions. 


\section{Acknowledgments}

We thank $\mathrm{Ch}$ McParland and $\mathrm{M}$ Bronson for constructing and helping us with the VME system, and G Krebs, F Lothrop and the BEVALAC team for providing an excellent $U$-beam and helping us set up the beam line. Important parts of the apparatus were transported from Frankfurt to San Francisco through the generosity of Lufthansa. Support for our work was provided by the Director, Office of Energy Research, Office for Basic Energy Sciences, Chemical Sciences Division, US Department of Energy, contract No DE-AC03-76SF00098 (MHP, HS-B, RD, JP), Kansas State University (SH, PR, HS-B), Office of Fusion Energy-US Department of Energy (REO) and the NATO Science Fellowship Program (JP). Additional support was provided by the Friedrich Ebert Stiftung (RD) and NATO research grant No 0501/85 (HB, CK, HS-B).

\section{References}

Cocke C L 1979 Phys. Rev. A 20749

Damsgaard D, Haugen H K, Hvelplund P and Knudsen H 1982 Phys. Rev. A 27172

Groh W, Müller A, Achenbach C, Schlachter A S and Salzborn E 1981 Phys. Lett. A 8577

Horbatsch M 1986 Z. Phys. D 1337

Horbatsch M and Dreizler R M 1986 Z. Phys. D 2183

Hvelplund P, Haugen H K and Knudsen H 1980 Phys. Rev. A 221930

Inokuti M 1971 Rev. Mod. Phys. 43279

Kelbch S, Ullrich J, Mann R, Richard P and Schmidt-Böcking H 1985 J. Phys. B: At. Mol. Phys. 18323

Kelbch S et al 1986 J. Phys. B: At. Mol. Phys. 19 L47

McParland $C$ and Bronson M 1987 Private communication

Müller A, Schuch B, Groh W, Salzborn E, Beyer H F, Mokler P H and Olson R E 1986 Phys. Rev. A 333010

Olson R E 1987 Private communication

Olson R E, Ullrich J and Schmidt-Böcking H 1987 J. Phys. B: At. Mol. Phys. 201809

Richard P, Ulirich J, Kelbch S, Schmidt-Böcking H, Mann R and Cocke C L 1985 Nucl. Instrum. Methods 240532

Schlachter A S, Berkner K H, Graham W G, Pyle R V, Schneider P J, Stalder K R, Stearns J W and Tanis J A 1981 Phys. Rev. A 232331

Ullrich J, Bethge K, Kelbch S, Schadt W, Schmidt-Böcking H and Stiebing K E 1986 J. Phys. B: At. Mol. Phys. 19437

Ullrich J, Cocke C L, Kelbch S, Mann R, Richard P and Schmidt-Böcking H 1984 J. Phys. B: At. Mol. Phys. $17 \mathrm{~L} 785$

Ullrich J, Horbatsch M, Dangendorf V, Kelbch S and Schmidt-Böcking H 1988 J. Phys. B: At. Mol. Opt. Phys. 21611

Ullrich J, Schmidt-Böcking H, Kelbch S, Berg H, Cocke C L, Hagmann S, Richard P, Schlachter A S and Mann R 1987 Nucl. Instrum. Methods B 23131 